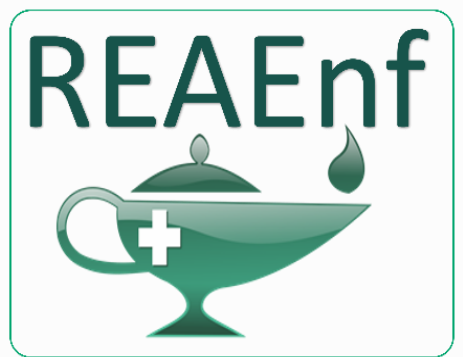

Revista Eletrônica Acervo Enfermagem
REVISÃO BIBLIOGRÁFICA

Recebido em: 6/2020

Aceito em: 7/2020

Publicado em: 9/2020

\title{
Aplicabilidade da cronofarmacologia no aprazamento de medicações em unidade de terapia intensiva: revisão integrativa
}

\author{
Chronopharmacology applicability in drug administration at intensive care unit: integrative \\ review
}

Aplicabilidad de la cronofarmacología en la administración de medicamentos en la unidad de cuidados intensivos: revisión integrativa

\begin{abstract}
Sibele Maria Schuantes Paim ${ }^{1 *}$, Daniela Couto Carvalho Barra², Gabriela Marcellino de Melo Lanzoni $^{2}$, Neide da Silva Knihs².
\end{abstract}

\begin{abstract}
Resumo: Identificar evidências científicas sobre a aplicabilidade da cronobiologia e cronofarmacologia para subsidiar a tomada de decisão clínica do enfermeiro no aprazamento das medicações potencialmente perigosas de pacientes internados em Unidade de Terapia Intensiva. Revisão integrativa da literatura desenvolvido em duas etapas: I) análise da lista de medicamentos dispensados para a Unidade de Terapia Intensiva; II) desenvolvimento das fases da revisão de literatura. As duas medicações mais dispensadas foram heparina e insulina. As evidências científicas apontaram que a heparina atinge o maior potencial terapêutico no período noturno. Em relação à insulina, não foi encontrado estudo sobre sua administração exógena e os achados científicos evidenciaram desacordo quanto ao ritmo circadiano da concentração plasmática da insulina e a responsividade das células B pancreáticas. Porém, estudos apontaram que a sensibilidade à insulina é menor durante a noite. Os dados encontrados são pioneiros e podem subsidiar o enfermeiro no que tange sua prática de aprazamento de medicações em Unidades de Terapia Intensiva. Tais achados podem contribuir para que os medicamentos sejam administrados em momentos adequados, visando à maximização das suas indicações clínicas junto aos pacientes em cuidados intensivos.
\end{abstract}

Palavras-chave: Esquema de medicação, Fenômenos cronobiológicos, Enfermagem.

\begin{abstract}
To identify scientific evidences about applicability of chronobiology and chronopharmacology to subsidize nurse decision making in potentially hazardous drugs administration at Intensive Care Unit. Literature integrative review developed in two steps: I) analysis of drug list dispensed to Intensive Care Unit; II) literature integrative review. Heparin and insulin were the drugs most dispensed. The scientific evidences show that heparin have the bigger therapeutic potential at night. About insulin, the selected studies don't approach the exogen insulin administration. These evidences disagree about the circadian rhythm, plasmatic concentration and B cells responsiveness. However, they show that insulin sensibility it's smaller at night. These data are pioneering and can subsidize nurses in the drug administration practice at Intensive Care Units. Evidences can contribute for better time management of drugs administration, aiming the maximization of clinicals indications at intensive care patients.
\end{abstract}

Keywords: Drug administration schedule, Chronobiology phenomena, Nursing.

1 Universidade Federal de São Paulo (UNIFESP), São Paulo - SP. *E-mail: sibele.schuantes@hotmail.com

2 Universidade Federal de Santa Catarina (UFSC), Florianópolis - SC. 
Resumen: Identificar evidencia científica sobre la aplicabilidad de la cronobiología y la cronofarmacología para apoyar la toma de decisiones clínicas de las enfermeras en la programación de medicamentos potencialmente peligrosos a los pacientes ingresados en la Unidad de Cuidados Intensivos. Revisión integral de la literatura desarrollada en dos etapas: I) análisis de la lista de medicamentos dispensados a la Unidad de Cuidados Intensivos; II) desarrollo de las fases de revisión de la literatura. Los dos medicamentos más dispensados fueron la heparina y la insulina. La evidencia científica ha revalo que la heparina alcanza su mayor potencial terapéutico en la noche. Con respecto a la insulina, no se encontró ningún estudio sobre su administración exógena y los hallazgos científicos mostraron desacuerdo con respecto al ritmo circadiano de la concentración de insulina en plasma y la capacidad de respuesta de las células B pancreáticas. Sin embargo, los estudios han demostrado que la sensibilidad a la insulina es menor por la noche Tales hallazgos pueden contribuir a que la medicación se administre en los momentos apropiados, con el objetivo de maximizar sus indicaciones clínicas con pacientes en cuidados intensivos.

Palabras clave: Esquema de medicación, Fenómenos cronobiológicos, Enfermería.

\section{INTRODUÇÃO}

$\mathrm{Na}$ área de pesquisa em saúde encontram-se inúmeros conceitos. Um deles que ganhou destaque no ano de 2017 foi o de cronobiologia. Três pesquisadores receberem o prêmio Nobel de fisiologia ao divulgarem suas descobertas relacionadas aos mecanismos moleculares responsáveis por determinar 0 ritmo circadiano (MCKENNA HT, et al., 2017).

A Cronobiologia refere-se ao estudo dos ritmos diários, variações associadas à temperatura e secreção hormonal, por exemplo, que são responsáveis pela manutenção da homeostase. A cronofarmacologia é uma área específica da cronobiologia, que investiga a associação de tais variações biológicas com a farmacodinâmica (mecanismos de ação, efeitos desejados ou colaterais) e farmacocinética (características relacionadas com absorção, distribuição e eliminação dos metabólitos). Seu objetivo principal é a obtenção da melhor ação terapêutica dos fármacos respeitando os ritmos diários já existentes (TAHARA Y e SHIBAT, 2014; PANDI-PERUMAL SR, et al., 2008).

No que diz respeito à administração de medicamentos, a enfermagem vivencia um contínuo desafio para desenvolver práticas que evitem os riscos relacionados à assistência à saúde. Em concordância a isso, a Organização Mundial da Saúde (OMS) lançou em 2004 a Aliança Mundial para a Segurança do Paciente e, há três anos, o Terceiro Desafio Global para a Segurança do Paciente, com a pretensão de reduzir em 50\% os erros relacionados à medicação (MINISTÉRIO DA SAÚDE, 2014; OPAS, 2017; OPAS, 2019).

Nesse contexto de incentivo e fomento à prática segura na atenção ao paciente, realidades como a das Unidades de Terapia Intensiva (UTIs), que são cenários de atendimento altamente especializado com ênfase no tratamento tecnológico e poli medicamento, são alvo para estratégias que subsidiem um cuidado cada vez mais seguro (LLAPA-RODRIGUEZ EO, et al., 2017; OLIVEIRA JKA, et al., 2018).

Diante de tal cenário, o enfermeiro exerce atividade fundamental e intimamente relacionada à prática segura, nomeadamente a prática em relação aos medicamentos. Este profissional é responsável pelo aprazamento, preparo, administração e checagem, ou seja, todo o processo desde o recebimento do receituário até a manutenção do paciente. Uma dessas responsabilidades é a determinação dos horários em que cada medicação será administrada, ou seja, o esquema de medicação ou aprazamento. É nesse momento em que se sugere a aproximação deste profissional com os conceitos científicos de cronofarmacologia, uma vez que esse procedimento se baseia normalmente, em rotinas hospitalares (LLAPA-RODRIGUEZ EO, et al., 2017; SILVA DS, et al., 2013).

No ambiente poli medicamentoso de uma UTI há outros conceitos intrinsecamente relacionados à segurança do paciente, particularmente os Medicamentos Potencialmente Perigosos (MPP). Tal conceito foi criado pelo Institute for Safe Medication Practices (ISMP) e visa classificar alguns medicamentos que apresentam risco significativo de causar sérios danos aos pacientes quando administrados desnecessário 
ou erroneamente. Quando os pacientes são expostos a erros relacionados a tais medicamentos o dano pode ser fatal. Destaca-se, portanto, a exigência de alta vigilância a fim de evitar a ocorrência de eventos adversos relacionados a essas medicações, como por exemplo, quimioterápicos, insulina, bloqueadores neuromusculares, anticoagulantes, entre outros (ISMP, 2018).

Assim, este estudo visa responder a seguinte questão de pesquisa: Quais são as evidências científicas sobre a aplicabilidade da cronobiologia e cronofarmacologia que podem subsidiar a tomada de decisão do enfermeiro em relação ao aprazamento de Medicamentos Potencialmente Perigosos em Unidades de Terapia Intensiva?

O objetivo da pesquisa é identificar evidências científicas sobre a aplicabilidade da cronobiologia e cronofarmacologia para subsidiar a tomada de decisão clínica do enfermeiro no aprazamento das medicações potencialmente perigosas de pacientes internados em Unidade de Terapia Intensiva.

\section{MÉTODOS}

Trata-se de um estudo de revisão de literatura realizado a partir de duas etapas distintas: Etapa I seleção das medicações a serem pesquisadas a partir da lista de medicamentos dispensados para uma UTI com dez leitos ativos, de um hospital de grande porte da região sul do Brasil; Etapa II - conforme as fases que compõem um estudo de revisão de literatura (GANONG LH, 1987).

Para a Etapa I, a identificação e seleção das medicações, buscou-se o serviço de almoxarifado da referida instituição e solicitou-se a listagem das medicações dispensadas para a UTI no decorrer de nove meses, ou seja, de janeiro a setembro de 2018. Após uma leitura minuciosa da listagem, realizada em conjunto com um farmacêutico clínico, incluiu-se como critério para seleção das medicações: Medicamentos Potencialmente Perigosos (MPP) com o maior número de dispensações para o setor.

Destaca-se que, como o estudo buscou identificar as evidências científicas sobre a aplicabilidade da cronobiologia e a cronofarmacologia aos MPP, os medicamentos administrados por via endovenosa em bomba de infusão contínua foram excluídos desta pesquisa.

A Etapa II ocorreu a partir do desenvolvimento das fases que compõem a uma revisão integrativa da literatura, assim especificadas: apresentação da questão de pesquisa e objetivo da pesquisa, busca da literatura, avaliação dos dados, análise dos dados e apresentação dos resultados (GANONG LH, 1987).

Para a busca das evidências científicas foram selecionadas as bases de dados, bem como, construídas as estratégias de busca para cada medicação e critérios de inclusão e exclusão dos estudos. Para cada uma das medicações selecionadas foi realizado um processo individual de revisão.

As bases de dados pesquisadas foram Web of Science, US National Library of Medicine and National Institutes of Health (PubMed), SCOPUS; Literatura Latino-americana e do Caribe em Ciências da Saúde (LILACS) e Embase. Ressalta-se que a busca realizada nas diversas bases visou a ampliar as possibilidades de se acessar estudos que contemplassem o escopo da pesquisa. A pesquisa nas bases de dados foi realizada no período de janeiro a maio de 2019.

As medicações selecionadas para o estudo foram: 1) heparina: apresenta ação anticoagulante, sendo considerada MPP em 96\% de risco (ISMP, 2018; JUNQUEIRA DRG, et al., 2011) dispensada 3.541 vezes pelo almoxarifado hospitalar e; 2) insulina humana Neutral Protamine Hagedorn (NPH) e Regular: apresenta ação hipoglicemiante e é considerada MPP em 98\% de risco (ISMP, 2018); dispensada 69 vezes no período de nove meses listados.

As estratégias de busca combinadas com operadores booleanos para a revisão em cada base de dados sobre heparina foram as seguintes: ((Heparin) OR Heparin treatment) AND Chronobiology Phenomena / ((heparin) OR (heparin AND treatment) AND (circadian AND rhythm)) / TÓPICO: (heparin) AND TÓPICO: (Chronobiology) / TÓPICO: (heparin) AND TÓPICO: (Circadian rhythm) / (Heparin) AND TÓPICO: (Chronobiology Phenomena) OR TÓPICO: (Chronobiology Discipline) OR TÓPICO: (Circadian rhythm) / 
heparina [Palavras] and fenômenos Cronobiológicos [Descritor de assunto] or ritmo circadiano [Descritor de assunto] / heparin AND chronobiology AND ('circadian rhythm'/exp OR 'circadian rhythm').

As estratégias de buscas para insulina foram: ((insulin) AND chronobiology phenomena) AND chronobiology /(insulin) AND (chronobiology AND phenomena)) / TÓPICO: (insulin) AND TÓPICO: (Chronobiology) / insulina [Palavras] and ritmo circadiano [Descritor de assunto] / ('insulin'/exp OR insulin) AND chronobiology OR 'circadian rhythm').

Critérios de inclusão: estudos com seres humanos, publicados nos últimos cinco anos, nos idiomas português, inglês e espanhol, que compreendessem a relação entre a medicação estudada e os conceitos de cronobiologia e/ou cronofarmacologia. Vale ressaltar que na primeira busca, ao incluir o filtro "últimos cinco anos", houve escassez de estudos publicados. Assim, optou-se por reiniciar a busca e não incluir o limite temporal para esta pesquisa. Critérios de exclusão: artigos de revisão, editoriais, cartas e resumos em anais de eventos, estudos na área pediátrica, estudos in vitro e estudos indisponíveis na íntegra mesmo após contato com o pesquisador responsável ou ainda, estudos em idioma diferente dos estabelecidos.

A fim de organizar a seleção dos trabalhos encontrados, utilizou-se do software gerenciador de referências Mendeley®.

A seleção e análise dos trabalhos seguiu individualmente o seguinte método: 1) busca dos estudos nas bases de dados com as estratégias de busca; 2) coleta dos dados dos estudos, considerando as características comuns e necessárias com base na leitura de título e resumo; 3 ) análise criteriosa dos estudos incluídos por meio da leitura na íntegra, considerando os aspectos relativos à medicação e a cronofarmacologia, assim como classificação de acordo com o nível de evidência; 4) interpretação e discussão dos resultados; 5) apresentação da revisão integrativa de forma a construir o conhecimento e contribuir para o cenário científico (GANONG LH, 1987; MOHER D, et al., 2010).

Para a classificação dos níveis de evidência dos estudos selecionados para análise utilizou-se a proposição do Joana Briggs Institute (2014).

A fim de sistematizar ainda mais os resultados, um fluxograma PRISMA foi apresentado. Tal modelo permite visualizar os estudos identificados em cada base de dados, assim como, os estudos excluídos, visualizando-se o total de artigos estudados que embasaram o atual estudo (MOHER D, et al., 2010).

\section{RESULTADOS E DISCUSSÃO}

Os estudos identificados e selecionados para a revisão são apresentados conforme o modelo PRISMA (Figura 1).

Em relação à heparina, encontrou-se 784 estudos utilizando as estratégias de busca nas bases de dados. Aplicando os critérios de inclusão/exclusão, 34 estudos foram pré-selecionados após a leitura de título e resumo. Em seguida, prosseguiu-se com a leitura na íntegra dos artigos, resultando em 4 estudos que cumpriram os todos os critérios estabelecidos para análise. Os 4 artigos selecionados são dos seguintes países: Países Baixos, França, Estados Unidos e Suécia; e foram publicados nos anos de 1985, 1989, 1994 e 2001, respectivamente.

Quanto ao MPP insulina, após a busca nas bases de dados, identificou-se 411 artigos, destes 55 foram pré-selecionados após a leitura de títulos e resumos. Com a leitura na íntegra, foram selecionados 5 estudos para análise das evidências científicas. Dois dos estudos selecionados foram publicados em 2016, e os demais foram em 2012, 2013 e 2018. Os países de origem das pesquisas foram Estados Unidos da América (1), Itália (2), Singapura (1) e Espanha (1). 
Figura 1 - Estudos selecionados para revisão de literatura.
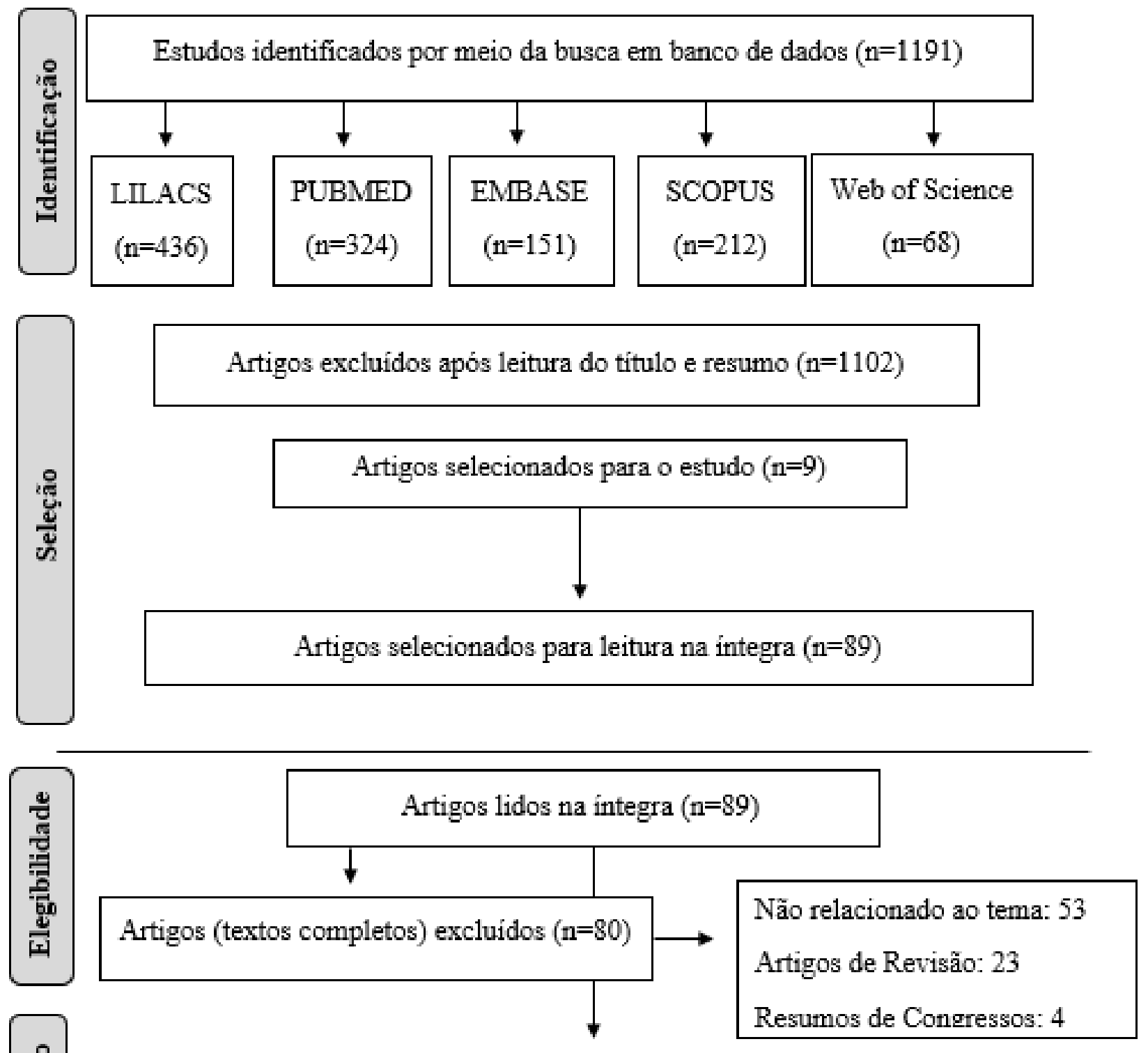

Artigos selecionados para o estudo (n=9)

Fonte: Paim SMS, et al., 2020.

A relação de título, autoria, tipo de estudo, nível de evidência e objetivo do estudo das medicações mencionadas estão demonstrados abaixo (Quadro 1). 
Quadro 1 - Artigos selecionados para revisão dos MPP Heparina e Insulina: título do artigo, autoria, tipo de estudo, nível de evidência (NE) e objetivo do estudo. Florianópolis-SC, 2019.

\begin{tabular}{|c|c|c|}
\hline \multicolumn{3}{|c|}{ Heparina } \\
\hline Autores & $\begin{array}{c}\text { Tipo do Estudo e Nível de } \\
\text { Evidência (NE) }\end{array}$ & Objetivo do Estudo \\
\hline DECOUSUS HA, et al., 1985 & $\begin{array}{l}\text { Estudo clínico } \\
\text { NE: } 4\end{array}$ & $\begin{array}{l}\text { Identificar a variação circadiana da ação } \\
\text { anticoagulante da heparina infundida } \\
\text { durante } 48 \text { horas em pacientes com } \\
\text { tromboembolismo venoso. }\end{array}$ \\
\hline FAGRELL B, et al, 1989 & $\begin{array}{l}\text { Estudo clínico } \\
\text { NE: } 4\end{array}$ & $\begin{array}{l}\text { Testar as variações circadianas jáa } \\
\text { apresentadas por um estudo Francês numa } \\
\text { amostra da população da Suécia. }\end{array}$ \\
\hline KRULDER JWM, et al., 1994 & $\begin{array}{l}\text { Acompanhamento } \\
\text { de } 24 \text { horas } \\
\text { NE: } 4\end{array}$ & $\begin{array}{l}\text { Avaliar a importância das variações diurnas } \\
\text { no efeito de infusão contínua e não } \\
\text { fracionada de heparina em pacientes com } \\
\text { tromboembolismo venoso. }\end{array}$ \\
\hline HODOGLUGIL U, et al., 2001 & $\begin{array}{l}\text { Estudo clínico } \\
\text { NE: } 4\end{array}$ & $\begin{array}{l}\text { Compreender se } \\
\text { variações circanuais referente ao uso de } \\
\text { heparina na homeostasia sanguínea para } \\
\text { cirurgia cardíaca. }\end{array}$ \\
\hline \multicolumn{3}{|c|}{ Insulina } \\
\hline QIAN J, et al., 2018 & $\begin{array}{l}\text { Design cruzado, randomizado, } \\
\text { com dois protocolos de } \\
\text { laboratório de oito dias } \\
\text { NE: } 3\end{array}$ & $\begin{array}{l}\text { Compreender os efeitos do sistema } \\
\text { circadiano e do desalinhamento circadiano } \\
\text { em relação a sensibilidade à insulina e à } \\
\text { secreção de insulina em humanos. }\end{array}$ \\
\hline CHUA ECP, et al., 2013 & $\begin{array}{c}\text { Design cruzado, randomizado, } \\
\text { com protocolo de laboratório de } \\
\text { quatro dias } \\
\text { NE: } 3\end{array}$ & $\begin{array}{l}\text { Identificar a variação circadiana de lipídeos } \\
\text { no plasma sanguíneo. }\end{array}$ \\
\hline SAAD A, et al., 2012 & $\begin{array}{l}\text { Estudo cruzado de três } \\
\text { tratamentos (refeições) em três } \\
\text { períodos (dias de estudo) } \\
\text { NE: } 3\end{array}$ & $\begin{array}{l}\text { Determinar se existem alterações diurnas } \\
\text { em relação a tolerância a glicose pós- } \\
\text { prandial, ação da insulina, secreção da } \\
\text { insulina e apresentação da glicose nas } \\
\text { refeições em indivíduos não diabéticos } \\
\text { usando a técnica do triplo-marcador. }\end{array}$ \\
\hline $\begin{array}{c}\text { CARRASCO-BENSO MP, et } \\
\text { al., } 2016\end{array}$ & $\begin{array}{l}\text { Estudo clínico } \\
\text { NE: } 4\end{array}$ & \begin{tabular}{|lcr} 
Investigar se & 0 tecido & adiposo \\
humano expressa & ritmos & circadianos \\
intrínsecos a sensibilidade a insulina que \\
poderiam contribuir para este fenômeno.
\end{tabular} \\
\hline MORRIS C, et al., 2016 & \begin{tabular}{|} 
Estudo randomizado, cruzado, \\
com duas visitas laboratoriais de \\
três dias \\
NE: 3
\end{tabular} & 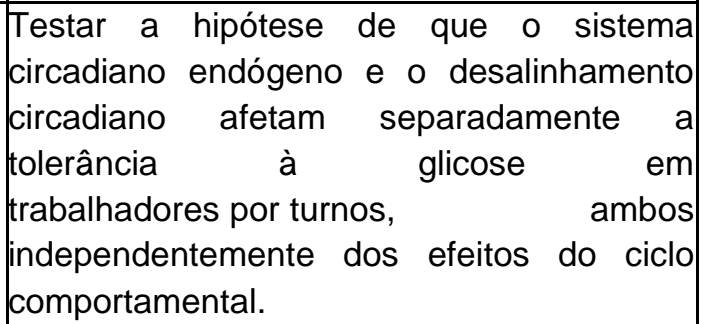 \\
\hline
\end{tabular}

Fonte: Paim SMS, et al., 2020.

A maior complicação relacionada ao uso de heparina é o sangramento, por se tratar de uma medicação com função anticoagulante. Portanto, aspectos como manutenção da dosagem, observação crítica dos exames de coagulação e exame físico do paciente, torna-se fundamental para evitar um desfecho crítico para o paciente (MULLOY B, et al., 2015; ODUAH E, et al., 2016; HODOGLUGIL U, et al., 2001). 
Dos estudos citados, dois deles detectaram que o pico da ação anticoagulante da heparina ocorre no período noturno/madrugada, entre quatro a seis horas; e a menor ação terapêutica ocorre no período da manhã, com pico às 12 horas (DECOUSUS HA, et al., 1985; KRULDER JWM, et al., 1994). No entanto, os pesquisadores não indicam mudanças na prática clínica imediata, mas relatam que os resultados permitem que a administração da heparina seja ajustada nos horários de melhor ação terapêutica.

Os dados revelaram que a perfusão contínua da medicação não significa a manutenção da ação terapêutica, ou seja, pode haver hiperdosagem a noite e subdosagem durante a manhã (DECOUSUS HA, et al., 1985; FAGRELL B, et al, 1989).

Esses fatores colocam esta medicação na lista dos Medicamentos Potencialmente Perigosos com 96\% de risco e estratégias de promover a acurácia na forma de administração tornam-se extremamente importantes no que diz respeito à segurança do paciente (ISMP, 2018). No transcorrer da análise dos manuscritos originais quanto ao uso de insulina, compreende-se que os achados podem contribuir de maneira significativa para as discussões clínicas e a tomada de decisão do enfermeiro referente ao melhor momento para a administração da insulina, assim como, subsidiar estudos futuros sobre a cronofarmacologia aplicada à administração desse medicamento.

Apesar das pesquisas selecionadas para análise serem publicações mais recentes, é possível encontrar achados contraditórios em cada estudo. O pico de concentração plasmático de insulina, determinado em um estudo realizado com 20 participantes homens saudáveis sob condições controladas em um laboratório, e com exames de sangue sendo coletados a cada quatro horas, evidenciou que a maior concentração ocorre entre o momento de acordar e o horário do almoço (CHUA ECP, et al., 2013). Por outro lado, outro trabalho com 20 participantes homens e mulheres saudáveis em condições controladas, detectou o maior pico no horário do jantar (SAAD A, et al., 2012).

Outros autores corroboraram com os seus achados, evidenciando que a meia noite é o horário em que há menor sensibilidade à insulina e ao meio dia é o pico de sensibilidade. No entanto, os resultados do estudo foram obtidos através de biópsias de tecido adiposo subcutâneo e visceral de 18 indivíduos que estavam prestes a fazer cirurgia gástrica. A amostra dos participantes era composta por diabéticos (nove) e não diabéticos (nove), e no dia anterior à cirurgia, todos fizeram as mesmas refeições nos mesmos horários. Ou seja, os autores identificaram o padrão de menor sensibilidade à noite, evidenciando que neste horário os níveis de glicose serão mais dificilmente normalizados (CARRASCO-BENSO MP, et al., 2016).

Associado ao pico de insulina plasmático e a sensibilidade à insulina, outro fator que foi apresentado pelos estudos foi a responsividade das células beta-pancreáticas, responsáveis pela produção e liberação de insulina. Neste aspecto houve também discordâncias entre os estudos encontrados. Dois deles evidenciaram a melhor responsividade dessas células no período noturno (QIAN J, et al., 2018; MORRIS C, et al., 2016). Enquanto outro apontou no período da manhã (SAAD A, et al., 2012).

Com os dados apresentados, não é possível estabelecer um padrão circadiano que influencie a mudança clínica imediata do enfermeiro quanto ao aprazamento da insulina exógena, visto que os estudos ainda apresentam importantes contradições e métodos não comparáveis. No entanto, a presença da influência dos ritmos circadianos é perceptível, principalmente no que diz respeito às alterações que ocorrem diante do desalinhamento circadiano e devem seguir como base para discussões clínicas e desenvolvimento de estudos futuros.

O desalinhamento circadiano refere-se ao rompimento do ritmo endógeno do indivíduo por fatores externos como trabalho e por fatores internos como distúrbios circadianos (BARON KG e REID KJ, 2014). Dois estudos apresentaram evidências quanto aos efeitos relacionados ao desalinhamento circadiano. Identificaram menor sensibilidade à insulina e menor tolerância à glicose quando analisado trabalhadores em turnos, incluindo o maior risco desses trabalhadores em desenvolver síndromes metabólicas (QIAN J, et al., 2018; MORRIS C, et al., 2016).

Em todos os estudos analisados, os autores relataram a necessidade de maiores investigações para a definição dos padrões circadianos da insulina a fim de melhor combinar estratégias de cuidado a saúde às 
pessoas com síndromes metabólicas e também como forma de garantir melhor associação terapêutica exógena, combinando melhores dosagens em horários em que a insulina está em menor concentração no plasma sanguíneo, evitando superdosagens em horários de maior sensibilidade à insulina (QIAN J, et al., 2018; CHUA ECP, et al., 2013; MORRIS C, et al., 2016).

Vale ainda pontuar, que a insulina é amplamente utilizada em UTIs visto que é uma estratégia para a estabilização do paciente crítico e é um Medicamento Potencialmente Perigoso com taxa de risco de $98 \%$. Os riscos associados à superdosagem deste medicamento podem gerar hipoglicemia, encefalopatia irreversível, edema pulmonar, danos hepáticos, coma hipoglicêmico e morte; já sua subdosagem pode causar hiperglicemia e cetoacidose (ISMP, 2012; RUSSELL KS, et al., 2009).

Este estudo traz um panorama geral sobre o uso dos conceitos de cronobiologia e cronofarmacologia no âmbito de dois Medicamentos Potencialmente Perigosos - heparina e insulina - todavia, não são suficientes para mudanças imediatas na prática clínica do enfermeiro quanto ao aprazamento dessas medicações. Destaca-se que a falta de estudos mais recentes nesta área, principalmente relacionados à cronofarmacologia em relação à administração de heparina e outras vias de administração dessa medicação, é uma limitação desta revisão.

O presente estudo teve como objetivo identificar as evidências científicas sobre cronobiologia e cronofarmacologia para subsidiar a tomada de decisão clínica do enfermeiro no aprazamento das medicações de pacientes internados em UTI. Destaca-se que as principais recomendações envolvem: que o horário em que a heparina atinge o maior potencial terapêutico é no período noturno. Não foi possível encontrar estudos referentes ao uso de insulina exógena relacionado à cronofarmacologia. Contudo, há concordância quanto ao ritmo circadiano da concentração plasmática da insulina e quanto a responsividade das células beta-pancreáticas. Ainda, foi evidenciado que a sensibilidade à insulina é menor durante a noite.

\section{CONSIDERAÇÕES FINAIS}

Diante de tais achados, fica evidente, que conceitos da aplicabilidade da cronofarnacolgia estão presentes e já foram divulgados, e devem ser levados em consideração nas discussões clínicas, uma vez que tratam de medicações em que a vigilância precisa ser constante. Os resultados deste estudo subsidiam o enfermeiro no que diz respeito a tomada de decisão clínica e ao raciocínio clínico apesar de serem necessários maiores aprofundamentos. É uma estratégia que pode evitar superdosagem ou subdosagem de medicações e, por conseguinte, complicações e eventos adversos advindos de cada uma delas. É importante o enfermeiro apropriar-se deste assunto e incorporar como estratégia de segurança do paciente.

\section{REFERÊNCIAS}

1. BARON KG, REID KJ. Circadian misalignment and health. International Review of Psychiatry, 2014; 26(2): $139-154$.

1. Ministério da Saúde. Documento de referência para o Programa Nacional de Segurança do Paciente. Brasília (DF): Ministério da Saúde; 2014.

2. CARRASCO-BENSO MP, et al. Human adipose tissue expresses intrinsic circadian rhythm in insulin sensitivity. The FASEB Journal, 2016; 30(9): 3117-3123.

3. CHUA ECP, et al. Extensive diversity in circadian regulation of plasma lipids and evidence for different circadian metabolic phenotypes in humans. PNAS, 2013; 110(35): 14468-14473.

4. DECOUSUS HA, et al. Circadian changes in anticoagulant effect of heparin infused at a constant rate. British Medical Journal (Clinical Research Ed.), 1985; 290(6465): 290-341.

5. FAGRELL B, et al. Changes of activated partial thromboplastin time during constant intravenous and fixed intermittent subcutaneous administration of heparin. Journal Of Internal Medicine, 1989; 225(4): 257-260.

6. GANONG LH. Integrative Reviews Lawrence of Nursing Research. Research in Nursing and Health, 1987; 10(1): 111.

7. HODOGLUGIL $U$, et al. Seasonal variation in the effect of a fixed dose of heparin on activated clotting time in patients prepared for open-heart surgery. Chronobiology International, 2001; 18(5): 195-203.

8. Institute for Safe Medication Practices (ISMP). Erros de medicação, riscos e práticas seguras na terpia com insulinas. Estados Unidos: ISMP; 2012. 
9. ISMP. 2018. In: High-Alert Medication Survey Results Lead to Several Changes for 2018. Disponível em: https://www.ismp.org/resources/high-alert-medication-survey-results-lead-several-changes-2018. Acesso em: 5 ago. 2020.

10. JUNQUEIRA DRG, et al. Farmacovigilância da heparina no Brasil. Rev Assoc Med Bras, 2011; 57(3): $328-332$.

11. KRULDER JWM, et al. Diurnal changes in heparin effect during continuous constant-rate infusion. A study in nine patients with venous thromboembolism. Journal Of Internal Medicine, 1994; 235(5): 411-417.

12. LLAPA-RODRIGUEZ EO, et al. Safe patient care in the preparation and administration of medicines. Revista Gaúcha de Enfermagem, 2017; 38(4): e2017-0029.

13. MCKENNA HT, et al. The significance of circadian rhythms and dysrhythmias in critical illness. Journal of Intensive Care Society, 2017; 18(2): 121-129.

14. MOHER D, et al. Preferred reporting items for systematic reviews and meta analyses: The PRISMA statement. International Journal of Surgery, 2010; 8(1): 336-341.

15. MORRIS C, et al. Effects of the Internal Circadian System and Circadian Misalignment on Glucose Tolerance in Chronic Shift Workers. The Journal of Clinical Endocrinology \& Metabolism, 2016; 101(3): 1066-1074.

16. MULLOY B, et al. Pharmacology of Heparin and Related Drugs. Pharmacological Reviews, 2015; 68(1): 76-141.

17. ODUAH E, et al. Heparin: Past, Present, and Future. Pharmaceuticals, 2016; 9(3): 38.

18. OLIVEIRA JKA, et al. Patient safety in nursing care during medication administration. Rev. Latino-Am. Enfermagem, 2018; 26(3): e3017.

19. OPAS. 2019 https://www.paho.org/bra/index.php?option=com_content\&view=article\&id=5384:omslanca-esforco-global-parareduzir-pela-metade-os-erros-relacionados-a-medicacao-em-cincoanos\&ltemid=838. Acesso em: 5 ago. 2020.

20. Organização Panamericana de Saúde (OPAS). Terceiro Desafio Mundial para Segurança do Paciente. Brasília (DF): OPAS; 2017.

21. PANDI-PERUMAL SR, et al. Sleep Disorders: Diagnosis and Therapeutics. Canadá: CRC Press; 2008; 153-162p.

22. QIAN J, et al. Differential effects of the circadian system and circadian misalignment on insulin sensitivity and insulin secretion in humans. Diabetes, Obesity and Metabolism, 2018; 20(10): 2481-2485.

23. RUSSELL KS, et al. Insulin Overdose Among Patients With Diabetes: A Readily Available Means of Suicide. The Primary Care Companion to the Journal of Clinical Psychiatry, 2009; 11(5): 258-262.

24. SAAD A, et al. Diurnal Pattern to Insulin Secretion and Insulin Action in Healthy Individuals. Diabetes, 2012; 61(11): 2691-2700.

25. SILVA DS, et al. Aprazamento de medicamentos por enfermeiros em prescrições de hospital sentinela. Texto contexto - enferm. 2013; 22(3): 722-730.

26. TAHARA Y, SHIBAT S. Chrono-biology, Chrono-pharmacology, and Chrono-nutrition. Journal of Pharmacological Sciences, 2014; 124(3): 320-335. 